\title{
Web Service-oriented Collaborative Management of Multi-Agent Supply and Demand Network
}

\author{
Zhou Li \\ Jinan Vocational College, Shandong, Jinan250000 \\ hunter2011@foxmail.com
}

Keywords: Web, Agent, Supply and demand network.

\begin{abstract}
The paper firstly introduces Web service technique, based on which the paper researches the supply and demand service under Web services-oriented supply and demand network collaborative management system architecture. Agent has features, so the paper focuses on researching integration method of Agent and Web service. The integration and interaction is collaborative. Agent makes Web service have intelligent feature, and can set intention and commitment according to the characteristics of tasks until the demand and supply service is satisfied. And Web service provides cross-platform interaction and sharing for Agent, which promotes the collaborative management of supply and demand network to be networked and intelligent.

Web service is a popular technique in information technique field in recent years, and it brings a new network application model. Web service technique is independent of the standard of software suppliers, and it implements cross-platform information system integration and data interaction with the loose coupling form. It is connected by Internet, and it provides service for other enterprises by the operation of release, discovery and binding. It can uniformly encapsulate all kinds of information, data and business process, and it can change the applications to be reusable and flexible components. Web service has the function of visiting enterprise business, which can dynamically discover and integrate supply and demand service of enterprises in real time and realize seamless interoperation of platform and operation system. And the enterprises can componentize core competence by abstracting and mixing, and the componentized core competence can be shared by different enterprises. Once the components based on Web service are deployed to the network, all applications accessing to the network can call and integrate Web services at any time, which not only helps the enterprises to discover or find useful services easily and rapidly, but also realizes the connection of business function of enterprises. Therefore, applying Web service technique to supply and demand network of enterprises not only optimizes the relationship between supply and demand service, but also finally realizes the objective of complementary advantages in resource and mutual benefit of supply and demand network node enterprises.
\end{abstract}

\section{Information Integration of Web Service-oriented Enterprise Supply and Demand Network}

Information integration of enterprise supply and demand network is the technical basis of implementing supply and demand network concept. It has the features of multi-function and openness, which drives the enterprise information systems to be integrated. Under the open environment, the changing information technique is applied to provide a completed information solution for system management system, software and hardware equipment and organizational management, and business process of enterprises. And the small operation environment of information islands of enterprises are integrated into a large operation environment to realize information sharing of supply and demand network. Applying Web service technique not only can make supply and demand network realize the integration of the existing systems easily, and integrates the new applications and connects all kinds of business processes, but also realizes the objective of appreciation and collaborative management of supply and demand network. 


\section{Web Service Technique}

Web service is the interface of business, application and system service based on XML standard. At present, the standards based on XML have been widely accepted, which is the key to realizing dynamic B2B business. The existing component techniques (such as DCOM) all use object model protocol, but Web service uses popular and open standard protocol such as HTTP and XML. Therefore, in the supply and demand network of enterprise researched in the paper, the standards of Web service which have been accepted are applied to provide supply and demand service for enterprises. In Web service, the provider of service can use WSDL to describe Web service, and can release and register Web service in UDDI registration center. The service requester query the service by UDDI, and the supply and requisitioning parties apply SOAP to bind and call service.

Web service system. Web service system structure explains how to instantiate elements and how to realize the operation with inter-operative form.

(1) Main roles of Web service system. Web service system includes three major roles, service provider, service requester and service registration center. Web service system structure is based on the interaction of three roles. And the interaction involves release, looking up and binding. During static binding development or dynamic binding execution, the service requester looks up the service and achieves the binding information of service (in service description). For static binding service requester, the service registration center is the optional role in architecture, the reason for which is that service provider can directly send the description to service requester. And the service requester can achieve service description from other resources except for service registration center such as local file, FIP site, Web site, advertisement and service discovery.

(2) Operation in Web service architecture. The applications using Web service must have the behaviors including releasing service description, querying or looking up service description and binding or calling service according to service description. The behaviors can appear once or repeatedly. And the operation are as follows.

Release. In order to make the service can be visited, the service description needs to be released to make service requester look up it. And the position of releasing service description can be changed according to the requirements of applications.

Look up. In look-up operation, the service requester directly searches service description or inquire the required service type in service registration center. In different life cycles, the service requester may involve look-up operation. During design, the interface description of service is searched for program development. During operation, the binding and position description of service is retrieved for calling.

Binding. Lastly, calling service is required. In binding operation, the service requester uses binding details of service description to locate, connect and call service, which can call or start the interaction with service in operation.

(3) Components of Web service. Service. Web service is an interface which is described by service description. And the implementation of service description is the service. Service is a software module. It is deployed on the platform which can be accessed by the network and is provided by service provider. The service can be called by service requester or interacted with service requester. When other Web services are used in service implementation, it can be used as requester.

Service description. Service description includes interface of service and implementation details. It not only includes data type of service, operation, binding information and network position, but also includes the classification and other metadata which can be discovered and used by the service requester easily. Service description can be released to service requester or service registration center.

Defining Web service--Web service description language (WSDL). In order to know where to deploy Web service in application of enterprises, and know what operations can be provided by the service, Web service must be described. It is WSDL(Web Services Description Language) that defines XML grammar, and describes what operations are completed by Web service to be the set 
of service access points making information exchange. It allows developers to make applications to be network service on Internet. In WSDL, the abstract definition of service access point and messages has been separated from concrete service deployment or data format binding, so the abstract definition can be reused. Message means abstract description for swap data, and port type means the abstract set of operations. The concrete protocols for specific port type and data format specifications compose the binding which can be reused. Correlating Web access address and reused binding can define a port, and the set of ports is defined as the service.

\section{Web service- oriented Collaborative Management System of Supply and Demand Network}

Framework of web service-oriented supply and demand. Supply and demand network system involves many enterprises such as supplier, manufacturer, retailer, transporter and client. And the development platforms and languages which are used by information system of enterprises may be different, which requires to integrate the applications which are developed by using different programming languages under different platforms to be a coordinated and interactive Web application. And Web service has advantages. It uses SOAP protocol to realize the communication between application and service, and WSDL file is used to describe service. Web service can be released on WEB, and the released service can be registered on UDDI for retrieval, which meets the loose coupling requirement of enterprises. According to the above design idea, the enterprise supply and demand network management system not only requires that supply and demand enterprises to make effective and rapid data exchange, but also needs to allow cooperation partners to inquire and search for some information of enterprises. And it can effectively integrate the existing systems of enterprises such as software of finance, and production and management.

Web service architecture and supply and demand service process. Web service includes three services, UDDI, service provider and service requester. In the supply and demand network environment, for supply and demand service, the physical architecture of supply service is similar to that of demand service, and only the process of dealing with service is different. Therefore, Web service system which can realize concrete supply and demand process not only can define Web service, inquire Web service and call Web service, but also can define, inquire and call enterprise supply and demand process.

\section{Method of Integrating Agent and Web Service in Supply and Demand Network}

Integrating Agent and Web service extends cooperation capabilities of supply and demand network. Integrating Agent and Web service extends collaborative management capabilities of supply and demand network, as follows.

(1) UDDI of a Web service only knows what service can be provided by it, but doesn't know the condition of other users. The autonomy, intelligence and mobility of Agent makes it have the capability of responding to the external and self perception. But Web service has no the perception capability and autonomy. It not only can't use other new capabilities of environment, but also can't reuse service actively according to the change of conditions. In the supply and demand environment, when an entity require Web service to provide customized product service, Web service gets the service request, Agent inquires the matched Agent of service provider according to the knowledge of knowledge base, and update the knowledge during interaction with Agent periodically. When there are the same service request, the Agent can use the updated knowledge to provide repeated service for service requester, which extends the capability of Web service greatly. Or, according to new service requests, Web service has no the capability of learning automatically, and can't update the existing knowledge, it only can recombine Web service, which influences the efficiency of Web service.

(2) The inherent communication capability of Agent makes the current information appear. It can actively send notification and update the existing information. But Web service is only triggered to provide service passively. The present standard and protocol can't provide a mechanism of updating service periodically. 
(3) Agent generally has an Ontologies. The interactive communication of Agent should obey the Ontologies, and Agents can be understood mutually. But Web service has no the common Ontologies. If the providers or requester of service use different Ontologies, Web service provides the service which can't be understood.

(4) Autonomy of Agent reflects that it is an entity which can provide belief, wish and commitment, which is very helpful for collaborative cooperation with other Agent or entities. By using the feature, it not only can ensure the communication of entity, but also can actively provide the commitment for other entities. But Web service has no the autonomy. Therefore, integrating Agent and Web service in demand and supply network can fully use automatic feature of Agent to provide high-level service for other entities.

Method of integrating Agent and Web service. According to the concept of Web service, from the perspective of service provider, if they can establish a Web site, they can connect the world. From the perspective of service requester, only clicking on internet can access to all registered tasks. The idea is consistent with the concept of supply and demand network. And the architecture of integrating Web service and Agent under the idea supports the implementation of collaborative management of supply and demand network. In the integration framework, task manager is the interface of the user with integration of Agent and Web service. Task manager accepts a task from the user, and establishes and manages a proper interface and initializes necessary resources. Agent server forms a working flow, and Agent executes tasks. In the process, task manager requires the users to provide constraints of resources, which is easy to compose working flow according to conditions.

Agent has the features of wish, intention and objective, so in the integrated structure, the communication between Agent and service should be based on a dialog protocol which means a standard and specification which is mutually obeyed. The process of communication is based on message interaction of dialogue. According to the characteristics of the structure, the information format includes the attributions of information and information content. Attribution is the header of message, and the content is the body of message.

Header:

From: address pf service requester

To: address pf service receiver

Protocol: name of protocol

Version: version of protocol

Content-Type: message type

Body: message content

The content of information consists of various information. The state of Agent reflects the communication and collaboration process. Therefore, the stage of Agent or service can consist of identification, objective (task), intention (for Agent), commitment (for a service) and knowledge (Agent or service).

\section{State_Agent/Service=\{ID_name, Goal, Intention, Commitments,Knowledge $\}$ ID_name $=\{$ Agent_name,Service_name $\}$}

(2) Knowledge $=\{$ info_1,info_2...info_n $\}$

(3) Goal= $=$ Form In,Form Out $\}$

If this is a service state, Form In describes precondition of service execution, and Form Out describes postposition result of service operation. If this is a state description of Agent, Form In may be empty or includes a format realizing precondition of Agent task, and Form Out describes task of Agent. (4) Commitments = \{Service_Commit1,Service_Commit2...Service_Commitn $\}$ Service_Commit $=\{$ forming,formOut $\}$ 
Form In is the precondition description of commitment, and Form Out is the result description of commitment implementation. When a commitment is realized, it is eliminated form commitment set, and the implementation process information is stored in knowledge base. (5) Intention $=\{$ Agent_Plans, Agent_Workflow, Agent_Realized $\}$

Plans is the set of describing Agent plan. Workflow is a series of flows of intentions. And the plans are some commitments relating to service acceptance plan. Realized is the flow of intentions beginning form working flow, and the working flow has realized commitment of service. In other words, the realization of intention of Agent is a series of process of state change.

\section{Conclusions}

Web service technique is independent of the standard of software supplier, and it realizes cross-platform information system integration and data interaction of enterprises.It can uniformly encapsulate all kinds of information, data and business process, and it can change the applications to be reusable and flexible components.Web service has the function of visiting enterprise business, which can dynamically discover and integrate supply and demand service of enterprises in real time. Once the components based on Web service are deployed to the network, all applications accessing to the network can call and integrate Web services at any time, which not only helps the enterprises to discover or find useful services easily and rapidly, but also realizes the connection of business function of enterprises.

Based on analyzing Web service technique, the paper researches architecture of web service-oriented supply and demand network collaborative management system, and researches the supply and demand collaboration service process under the structure. And the paper takes examples to research the design and development method of enterprise supply and demand Web service. Agent has features, so the paper focuses on researching the integration method of Agent and Web service. The method of integration Agent and Web service extends the functions of Web service, which can fully play the features of both in collaborative management of supply and demand network. Web service provides conditions for Agent to realize cross-platform communication. Agent technique provides intelligent feature for Web service technique. Therefore, the integration result of both promotes collaborative management of supply and demand network to be networked and intelligent.

\section{References}

[1] David Simchi-Levy, Philip Kaminsky, and Edith Simchi-Levy, Designing and Managing the Supply Chain, McGraw-Hill, 2000

[2] Hess, T., Rees, L, and Rakes, T., "Using Autonomous Software Agents to Create the Next Generation of Decision Support Systems,” Decision Sciences, Vol. 31, No. 1, 2000, pp. 1-31

[3] Ibrahim Cingil, An Architecture for Supply Chain Integration and Automation on the Internet, Distributed and Parallel Databases, Vol.10, 2001, pp.59-102

[4] J.X. Liu, S.S. Zhang and Jian Cao, An Inter-Enterprise Workflow Management System for B2B E-Commerce and Supply Chain, Systems, Man, and Cybernetics, 2001 IEEE International Conference on , Vol. 5, 2001, pp.2921 -2926

[5] Mark S.Fox, Mihai Barbuceanu, and Rune Teigen, Agent-oriented Supply-chain Management, The International Journal of Flexible Manufacturing Systems, Vol. 12, 2000, pp.165-188

[6] Qi Xu, Robin Qiu, and David Russell, Shop Floor Decision Support System using Multi-Agents, 6th World Multiconference on Systemics, Cybernetics and Informatics (SCI2002), Orlando, Florida, U.S.A, July 14-18, Vol.6, 2002, pp.475-479 
[7] Y.H. Yan, Zakaria Maamar and Weiming Shen, Integration of Workflow and Agent Technology for Business Process Management, Computer Supported Cooperative Work in Design, The Sixth International Conference on , 2001, pp.420 -426 